\title{
Dissipation energy in viscoelastic solids under multiaxial loads
}

\author{
Janusz Kolenda, \\ Naval Academy of Gdynia
}

\begin{abstract}

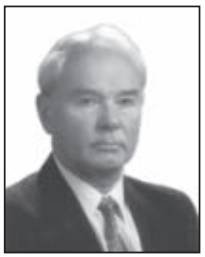

On the basis of the three-dimensional constitutive equations for strains resulting from the Kelvin-Voigt's model and modified Hooke's law for multiaxial stress in viscoelastic solids, the formulae for the energy dissipated in a given time per unit volume have been derived. It is shown that after application or removal of triaxial static load there is no difference in the time functions governing the dissipation of strain energy of volume change and the dissipation of strain energy of distortion. Harmonic in-phase stress and harmonic out-of-phase stress as well as multiaxial periodic stress are also considered. It is demonstrated that in the process of energy dissipation due to normal and shear stress components the role of the latter is dominant.
\end{abstract}

Keywords: viscoelastic material, multiaxial stress, energy dissipation, static load, vibratory load

\section{INTRODUCTION}

Engineering materials are not perfectly elastic and their service time periods may be relatively long. Therefore, at the design stage the energy dissipated through damping mechanisms in the material and, consequently, the rise of temperature of the parts loaded by time-varying forces, as well as the conditions of heat dissipation, are frequently taken into account. However, in practice such considerations are rarely followed by the estimation of amount of the energy dissipated in such parts in a given time. This situation may be avoided if an adequate damping model of the material and load history are known. As an example, in the present paper the KelvinVoigt's model of the material is used and the dissipation energy per unit volume as well as in a given volume is calculated in selected load cases. To simplify the calculations, the modified Hooke's law for multiaxial stress in viscoelastic solids [1] and the relevant three-dimensional constitutive equations for strains [2] are applied which requires that the material is homogeneous, isotropic and loaded below the yield point.

For the sake of clarity, our presentation of the problem solutions will start with some important relationships given in the literature.

\section{ENERGY DISSIPATED AFTER REMOVAL AND APPLICATION OF MULTIAXIAL STATIC LOAD}

If a viscoelastic rod is loaded for a long time by an axial force $\mathrm{P}=$ const, in its cross-section a uniformly distributed normal stress

$$
\sigma_{\mathrm{x} 0}=\frac{\mathrm{P}}{\mathrm{A}}
$$

is present, which corresponds to the normal strain:

$$
\varepsilon_{\mathrm{x} 0}=\frac{\sigma_{\mathrm{x}_{0}}}{\mathrm{E}}
$$

where:

A - area of the cross-section

E - Young modulus.

Removal of the load at the time $\mathrm{t}=0$ results in a creep recovery [2-4]:

$$
\varepsilon_{x}(t)=\varepsilon_{x 0} e^{-\frac{E}{\eta} t}
$$

where:

$\eta$ - coefficient of viscous damping of normal strain.

According to the Kelvin-Voigt's model of a viscoelastic material [3-5], the internal restoring force, $F$, and the counteracting resistance force, $\mathrm{R}$, can be expressed by:

$$
\begin{aligned}
& \mathrm{F}=-\mathrm{AE} \varepsilon_{\mathrm{x}} \\
& \mathrm{R}=\mathrm{A} \eta \dot{\varepsilon}_{\mathrm{x}}
\end{aligned}
$$

where the dot denotes differentiation with respect to $t$. Consequently, the dissipation energy can be calculated as the work, $\mathrm{W}$, of the restoring force. If at the time t the rod length is $1+\Delta 1$, where 1 is its length prior to load, then:

$$
\varepsilon_{\mathrm{x}}=\frac{\Delta 1}{1}
$$


and the elementary work of the restoring force is equal to

$$
\mathrm{dW}=\mathrm{Fd}(\Delta \mathrm{l})=\mathrm{Fld} \varepsilon_{\mathrm{x}}=\mathrm{Fl} \dot{\varepsilon}_{\mathrm{x}} \mathrm{dt}=-\mathrm{AEl} \varepsilon_{\mathrm{x}} \dot{\varepsilon}_{\mathrm{x}} \mathrm{dt}
$$

Denoting $\varphi$ the dissipation energy per unit volume, one obtains:

so that

$$
\mathrm{d} \phi=\frac{\mathrm{dW}}{\mathrm{Al}}=-\mathrm{E} \varepsilon_{\mathrm{x}} \dot{\varepsilon}_{\mathrm{x}} \mathrm{dt}
$$

$$
\phi(t)=-E \int_{0}^{t} \varepsilon_{x} \dot{\varepsilon}_{x} d t=\frac{E^{2}}{\eta} \varepsilon_{x_{0}}^{2} \int_{0}^{t} e^{-\frac{2 E}{\eta} t} d t
$$

Hence:

$$
\phi(t)=\frac{1}{2} E \varepsilon_{\mathrm{x}_{0}}^{2}\left(1-\mathrm{e}^{-\frac{2 \mathrm{E}}{\eta} \mathrm{t}}\right)=\frac{1}{2} \sigma_{\mathrm{x} 0} \varepsilon_{\mathrm{x} 0}\left(1-\mathrm{e}^{-\frac{2 \mathrm{E}}{\eta} \mathrm{t}}\right)
$$

and

$$
\lim _{\mathrm{t} \rightarrow \infty} \phi(\mathrm{t})=\frac{1}{2} \sigma_{\mathrm{x} 0} \varepsilon_{\mathrm{x} 0}
$$

Now let us consider what happens to a cubic element with fixed bottom side (Fig. 1) and subjected for a long time to the shear stress $\tau_{\mathrm{zy} 0}$ on its upper side, if at $\mathrm{t}=0$ the load is removed.

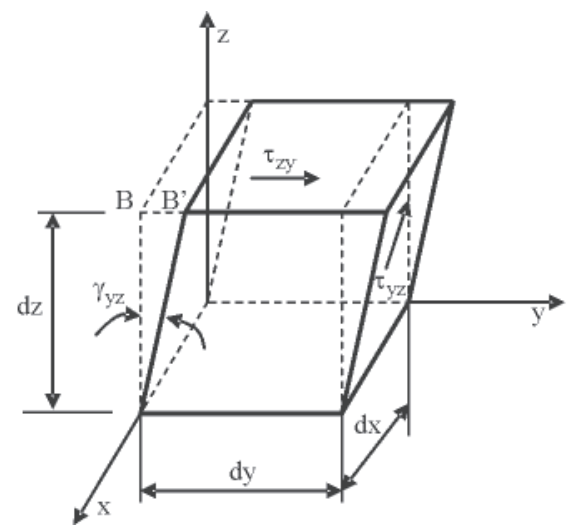

Fig. 1. Shear strain $\gamma_{y z}$ of infinitesimal cubic element

The restoring force and its elementary work on the line segment:

$$
\beta \beta^{\prime}=\gamma_{\mathrm{yz}} \mathrm{dz}
$$

are as follows:

$$
\mathrm{F}=-\mathrm{G} \gamma_{\mathrm{yz}} \mathrm{dxdy}
$$

$\mathrm{dW}=\mathrm{Fd} \gamma_{\mathrm{yz}} \mathrm{dz}=\mathrm{F} \dot{\gamma}_{\mathrm{yz}} \mathrm{dzdt}=-\mathrm{G} \gamma_{\mathrm{yz}} \dot{\gamma}_{\mathrm{yz}} \mathrm{dxdydzdt}$

where: $\gamma_{\mathrm{yz}}$ is the shear strain and

$$
\mathrm{G}=\frac{\mathrm{E}}{2(1+v)}
$$

is the shear modulus. Here $v$ is the Poisson's ratio. Thus:

$$
\mathrm{d} \phi=\frac{\mathrm{dW}}{\mathrm{dxdydz}}=-\mathrm{G} \gamma_{\mathrm{yz}} \dot{\gamma}_{\mathrm{yz}} \mathrm{dt}
$$

Knowing that removal of the shear load at $t=0$ is followed by the creep recovery [2]:

$$
\gamma_{y z}(t)=\gamma_{y z 0} e^{-\frac{E}{\eta} t}=\gamma_{y z 0} e^{-\frac{G}{\lambda} t}
$$

where: $\gamma_{\mathrm{yz} 0}=\tau_{\mathrm{yz} 0} / \mathrm{G}$ is the shear strain corresponding to the shear stress $\tau_{\mathrm{zy} 0}=\tau_{\mathrm{yz} 0}$, the dissipation energy per unit volume can be in this case expressed as:

$$
\phi(t)=-\mathrm{G} \int_{0}^{\mathrm{t}} \gamma_{\mathrm{yz}} \dot{\gamma}_{\mathrm{yz}} \mathrm{dt}=\frac{\mathrm{G}^{2}}{\lambda} \gamma_{\mathrm{yz} 0}^{2} \int_{0}^{\mathrm{t}} \mathrm{e}^{-\frac{2 \mathrm{G}}{\lambda} \mathrm{t}} \mathrm{dt}
$$

In Eqs (15) and (16), $\lambda$ is the coefficient of viscous damping of shear strain given by [2]:

$$
\lambda=\frac{\eta}{2(1+v)}
$$

Hence:

$\phi(t)=\frac{1}{2} G \gamma_{y z 0}^{2}\left(1-e^{-\frac{2 G}{\lambda} t}\right)=\frac{1}{2} \tau_{y z 0} \gamma_{y z 0}\left(1-e^{-\frac{2 E}{\eta} t}\right)$

and

$$
\lim _{t \rightarrow \infty} \phi(t)=\frac{1}{2} \tau_{y z 0} \gamma_{y z 0}
$$

It is noteworthy that the time function of energy dissipation by normal and shear stress components is the same.

Similar equations can be obtained for the dissipation energy in viscoelastic solids caused by the stress components $\sigma_{\mathrm{y} 0}, \sigma_{\mathrm{z} 0}, \tau_{\mathrm{xy} 0}$, and $\tau_{\mathrm{zx} 0}$ if at $\mathrm{t}=0$ a three-dimensional load has been removed. After summation of the resulting dissipation energies one gets:

where:

$$
\phi(t)=\psi_{0}\left(1-\mathrm{e}^{-\frac{2 \mathrm{E}}{\eta} \mathrm{t}}\right)
$$

$$
\begin{gathered}
\psi_{0}=\frac{1}{2}\left(\sigma_{\mathrm{x} 0} \varepsilon_{\mathrm{x} 0}+\sigma_{\mathrm{y} 0} \varepsilon_{\mathrm{y} 0}+\sigma_{\mathrm{z} 0} \varepsilon_{\mathrm{z} 0}+\right. \\
\left.+\tau_{\mathrm{xy} 0} \gamma_{\mathrm{xy} 0}+\tau_{\mathrm{yz} 0} \gamma_{\mathrm{yz} 0}+\tau_{\mathrm{zx} 0} \gamma_{\mathrm{zx} 0}\right)
\end{gathered}
$$

is the elastic strain energy per unit volume before removal of the load, and $[6,7]$ :

$$
\begin{aligned}
& \varepsilon_{\mathrm{x} 0}=\frac{1}{\mathrm{E}}\left[\sigma_{\mathrm{x} 0}-v\left(\sigma_{\mathrm{y} 0}+\sigma_{\mathrm{z} 0}\right)\right] \\
& \varepsilon_{\mathrm{y} 0}=\frac{1}{\mathrm{E}}\left[\sigma_{\mathrm{y} 0}-v\left(\sigma_{\mathrm{x} 0}+\sigma_{\mathrm{z} 0}\right)\right] \\
& \varepsilon_{\mathrm{z} 0}=\frac{1}{\mathrm{E}}\left[\sigma_{\mathrm{z} 0}-v\left(\sigma_{\mathrm{x} 0}+\sigma_{\mathrm{y} 0}\right)\right] \\
& \gamma_{\mathrm{k} 0}=\frac{1}{\mathrm{G}} \tau_{\mathrm{k} 0} ; \mathrm{k}=\mathrm{xy}, \mathrm{yz}, \mathrm{zx}
\end{aligned}
$$

are the strain components at $\mathrm{t}<0$.

With Eqs (22), $\psi_{0}$ can be expressed in terms of the stress components. It can be also divided into the strain energy of volume change, $\psi_{v 0}$, and strain energy of distortion, $\psi_{\mathrm{d} 0}$, that is:

$$
\psi_{0}=\psi_{\mathrm{v} 0}+\psi_{\mathrm{d} 0}
$$

where [6]:

$$
\begin{gathered}
\psi_{v 0}=\frac{1-2 \mathrm{v}}{6 \mathrm{E}}\left(\sigma_{\mathrm{x} 0}+\sigma_{\mathrm{y} 0}+\sigma_{\mathrm{z} 0}\right)^{2} \\
\psi_{\mathrm{d} 0}=\frac{1+\mathrm{v}}{6 \mathrm{E}}\left[\left(\sigma_{\mathrm{x} 0}-\sigma_{\mathrm{y} 0}\right)^{2}+\left(\sigma_{\mathrm{y} 0}-\sigma_{\mathrm{z} 0}\right)^{2}+\right. \\
\left.+\left(\sigma_{\mathrm{z} 0}-\sigma_{\mathrm{x} 0}\right)^{2}+6\left(\tau_{\mathrm{xy} 0}^{2}+\tau_{\mathrm{yz} 0}^{2}+\tau_{\mathrm{zx} 0}^{2}\right)\right]
\end{gathered}
$$


Eqs (20) and (23) lead to:

$$
\phi(t)=\left(\psi_{v 0}+\psi_{\mathrm{d} 0}\right)\left(1-\mathrm{e}^{-\frac{2 \mathrm{E}}{\eta} \mathrm{t}}\right)
$$

Hence it is clear that the time functions governing the dissipation of strain energy of volume change and the dissipation of strain energy of distortion are identical.

The problem of energy dissipation in viscoelastic materials after application of static loads can be solved analogously. For example, if an axial load is applied at $\mathrm{t}=0$ to a viscoelastic rod and the resulting stress $\sigma_{\mathrm{x} 0}$ is maintained constant, then a timedependent strain response will occur [2-4]

$$
\varepsilon_{\mathrm{x}}(\mathrm{t})=\varepsilon_{\mathrm{x} 0}\left(1-\mathrm{e}^{-\frac{\mathrm{E}_{\mathrm{f}}}{\eta}}\right)
$$

where: $\varepsilon_{\mathrm{x} 0}$ is given by Eq. (2). The dissipation energy in this case can be calculated as the work of this part of the external load which is overcoming the resistance force R.

The elementary work is then:

$$
\mathrm{dW}=\mathrm{Rd}(\Delta \mathrm{l})=\mathrm{R} 1 \mathrm{~d} \varepsilon_{\mathrm{x}}=\mathrm{R} 1 \dot{\varepsilon}_{\mathrm{x}} \mathrm{dt}=\mathrm{A} \ln \dot{\varepsilon}_{\mathrm{x}}^{2} \mathrm{dt}
$$

With Eqs (26) and (27a) one obtains:

and

$$
\mathrm{d} \phi=\frac{\mathrm{dW}}{\mathrm{Al}}=\eta \dot{\varepsilon}_{\mathrm{x}}^{2} \mathrm{dt}
$$

$$
\phi(t)=\frac{E^{2}}{\eta} \varepsilon_{x 0}^{2} \int_{0}^{t} e^{-\frac{2 E}{\eta} t} d t
$$

Hence the relationship for the dissipation energy per unit volume of the rod reads:

$$
\phi(\mathrm{t})=\frac{1}{2} \mathrm{E} \varepsilon_{\mathrm{x} 0}^{2}\left(1-\mathrm{e}^{-\frac{2 \mathrm{E}}{\eta} \mathrm{t}}\right)=\frac{1}{2} \sigma_{\mathrm{x} 0} \varepsilon_{\mathrm{x} 0}\left(1-\mathrm{e}^{-\frac{2 \mathrm{E}}{\eta} \mathrm{t}}\right)
$$

Of course,

$$
\lim _{t \rightarrow \infty} \phi(t)=\frac{1}{2} \sigma_{x 0} \varepsilon_{x 0}
$$

Note that the other part of the external constant load executes the work of elastic deformation of the rod which per unit volume is finally also equal to $1 / 2 \sigma_{x 0} \varepsilon_{x 0}[6]$. As a stored elastic strain energy, it will be dissipated after removal of the load [see Eq. (10)].

Similar equations can be written for the energy dissipated after application of the other stress components so that in the general case of static load applied at $t=0$ the summary dissipation energy per unit volume becomes again:

$$
\phi(t)=\psi_{0}\left(1-e^{-\frac{2 \mathrm{E}}{\eta} \mathrm{t}}\right)
$$

According to the Kelvin-Voigt's model,

$$
\mathrm{E} \varepsilon_{\mathrm{x}}+\eta \dot{\varepsilon}_{\mathrm{x}}=\sigma_{\mathrm{x}}
$$

so that the resistance force can be also expressed by

$$
\mathrm{R}=\mathrm{A} \eta \dot{\varepsilon}_{\mathrm{x}}=\mathrm{A}\left(\sigma_{\mathrm{x}}-\mathrm{E} \varepsilon_{\mathrm{x}}\right)
$$

Consequently, Eqs (27a) and (28a) can be rewritten as

$$
\begin{gathered}
\mathrm{dW}=\mathrm{A}\left(\sigma_{\mathrm{x}}-\mathrm{E} \varepsilon_{\mathrm{x}}\right) \mathrm{d}(\Delta \mathrm{l})=\mathrm{Al}\left(\sigma_{\mathrm{x}}-\mathrm{E} \varepsilon_{\mathrm{x}}\right) \dot{\varepsilon}_{\mathrm{x}} \mathrm{dt} \\
\mathrm{d} \phi=\left(\sigma_{\mathrm{x}}-\mathrm{E} \varepsilon_{\mathrm{x}}\right) \dot{\varepsilon}_{\mathrm{x}} \mathrm{dt}
\end{gathered}
$$

In what follows, Eq. (28b) and analogous relationships for the elementary dissipation energy due to the stress components $\sigma_{y}, \sigma_{z}, \tau_{x y}, \tau_{y z}$, and $\tau_{z x}$ will be applied.

\section{THE CASE OF HARMONIC IN-PHASE STRESS}

Suppose that a vibratory load is producing in a viscoelastic solid the stress of components:

$$
\begin{aligned}
& \sigma_{\mathrm{j}}=\sigma_{\mathrm{ja}} \sin \omega \mathrm{t} ; \mathrm{j}=\mathrm{x}, \mathrm{y}, \mathrm{z} \\
& \tau_{\mathrm{k}}=\tau_{\mathrm{ka}} \sin \omega \mathrm{t} ; \mathrm{k}=\mathrm{xy}, \mathrm{yz}, \mathrm{zx}
\end{aligned}
$$

where: $\sigma_{\mathrm{ja}}$ and $\tau_{\mathrm{ka}}$ are the amplitudes of the stress components and $\omega$ is their circular frequency. The relevant constitutive equation for strains reads [2]:

$$
\boldsymbol{\varepsilon}=\mathbf{H} \boldsymbol{\sigma}_{\mathrm{a}} \sin (\omega \mathrm{t}-\alpha) \varepsilon
$$

where $\boldsymbol{\varepsilon}$ is the vector of strain components. $\mathbf{H}$ is the matrix of dynamical flexibility of the material at the load circular frequency $\omega, \sigma_{\mathrm{a}}$ is the vector of amplitudes of the stress components and $\alpha$ is the phase shift between stress and strain components as follows:

$$
\begin{gathered}
\boldsymbol{\varepsilon}=\left[\varepsilon_{\mathrm{x}} \varepsilon_{\mathrm{y}} \varepsilon_{\mathrm{z}} \gamma_{\mathrm{xy}} \gamma_{\mathrm{yz}} \gamma_{\mathrm{zx}}\right]^{\mathrm{T}} \\
\boldsymbol{\sigma}=\frac{\sigma_{\mathrm{a}}=\left[\sigma_{\mathrm{xa}} \sigma_{\mathrm{ya}} \sigma_{\mathrm{za}} \tau_{\mathrm{xya}} \tau_{\mathrm{yza}} \tau_{\mathrm{zxa}}\right]^{\mathrm{T}}}{\sqrt{\mathrm{E}^{2}+\eta^{2} \omega^{2}}}\left[\begin{array}{cccccc}
1 & -v & -v & 0 & 0 & 0 \\
-v & 1 & -v & 0 & 0 & 0 \\
-v & -v & 1 & 0 & 0 & 0 \\
0 & 0 & 0 & 2(1+v) & 0 & 0 \\
0 & 0 & 0 & 0 & 2(1+v) & 0 \\
0 & 0 & 0 & 0 & 0 & 2(1+v)
\end{array}\right] \\
\alpha=\operatorname{arctg} \frac{\eta \omega}{\mathrm{E}}
\end{gathered}
$$


Starting with Eqs (28b), (34) and (35), for a viscoelastic cubic element under uniaxial stress:

$$
\sigma_{\mathrm{z}}=\sigma_{\mathrm{za}} \sin \omega \mathrm{t}
$$

we write:

$$
\begin{aligned}
& \mathrm{d} \phi=\left(\sigma_{\mathrm{z}}-\mathrm{E} \varepsilon_{\mathrm{z}}\right) \dot{\varepsilon}_{\mathrm{z}} \mathrm{dt} \\
& \varepsilon_{\mathrm{z}}=\frac{1}{\sqrt{\mathrm{E}^{2}+\eta^{2} \omega^{2}}} \sigma_{\mathrm{za}} \sin (\omega \mathrm{t}-\alpha)
\end{aligned}
$$

Thus, during the stress period $T=2 \pi / \omega$ the amount of dissipated energy per unit volume, $\varphi(T)$, becomes:

Obviously,

$$
\phi(T)=\int_{0}^{T}\left(\sigma_{z}-E \varepsilon_{z}\right) \dot{\varepsilon}_{z} d t
$$

which leads to:

$$
\int_{0}^{\mathrm{T}} \varepsilon_{z} \dot{\varepsilon}_{z} \mathrm{dt}=0
$$

$$
\phi(T)=\int_{0}^{T} \sigma_{z} \dot{\varepsilon}_{z} d t=\frac{1}{2} \frac{\omega T \sin \alpha}{\sqrt{E^{2}+\eta^{2} \omega^{2}}} \sigma_{z a}^{2}
$$

In the case of harmonic shear stress:

$$
\tau_{\mathrm{yz}}=\tau_{\mathrm{yza}} \sin \omega \mathrm{t}
$$

in place of Eq. (28b) we have:

$$
\mathrm{d} \phi=\left(\tau_{\mathrm{yz}}-\mathrm{G} \gamma_{\mathrm{yz}}\right) \dot{\gamma}_{\mathrm{yz}} \mathrm{dt}
$$

Eqs (34) and (35) yield:

$$
\gamma_{\mathrm{yz}}=\frac{2(1+v)}{\sqrt{\mathrm{E}^{2}+\eta^{2} \omega^{2}}} \tau_{\mathrm{yza}} \sin (\omega \mathrm{t}-\alpha)
$$

Accordingly,

$$
\phi(\mathrm{T})=\int_{0}^{\mathrm{T}} \tau_{\mathrm{yz}} \dot{\gamma}_{\mathrm{yz}} \mathrm{dt}=\frac{(1+v) \omega \mathrm{T} \sin \alpha}{\sqrt{\mathrm{E}^{2}+\eta^{2} \omega^{2}}} \tau_{\mathrm{yza}}^{2}
$$

If the viscoelastic element is simultaneously subjected to the stress components (36) and (41), then:

$$
\phi(T)=\frac{1}{2} \frac{\omega \mathrm{T} \sin \alpha}{\sqrt{\mathrm{E}^{2}+\eta^{2} \omega^{2}}}\left[\sigma_{\mathrm{za}}^{2}+2(1+v) \tau_{\mathrm{yza}}^{2}\right]
$$

Summarizing, the following formula for the dissipation energy per unit volume during the period $\mathrm{T}$ can be written:

$$
\phi(\mathrm{T})=\int_{0}^{\mathrm{T}}\left(\sigma_{\mathrm{x}} \dot{\varepsilon}_{\mathrm{x}}+\sigma_{\mathrm{y}} \dot{\varepsilon}_{\mathrm{y}}+\sigma_{\mathrm{z}} \dot{\varepsilon}_{\mathrm{z}}+\tau_{\mathrm{xy}} \dot{\gamma}_{\mathrm{xy}}+\tau_{\mathrm{yz}} \dot{\gamma}_{\mathrm{yz}}+\tau_{\mathrm{zz}} \dot{\gamma}_{\mathrm{zx}}\right) \mathrm{dt}
$$

With Eqs (33) through (35), one obtains from Eq. (46).

$$
\begin{aligned}
\phi(\mathrm{T})=\frac{1}{2} \frac{\omega \mathrm{T} \sin \alpha}{\sqrt{\mathrm{E}^{2}+\eta^{2} \omega^{2}}} & {\left[\sigma_{\mathrm{xa}}^{2}+\sigma_{\mathrm{ya}}^{2}+\sigma_{\mathrm{za}}^{2}-2 v\left(\sigma_{\mathrm{xa}} \sigma_{\mathrm{ya}}+\sigma_{\mathrm{ya}} \sigma_{\mathrm{za}}+\sigma_{\mathrm{za}} \sigma_{\mathrm{xa}}\right)+\right.} \\
& \left.+2(1+v)\left(\tau_{\mathrm{xya}}^{2}+\tau_{\mathrm{yza}}^{2}+\tau_{\mathrm{zxa}}^{2}\right)\right]
\end{aligned}
$$

Eqs (45) and (47) show that the shares of normal and shear stress components in the summary dissipation energy may be different. In order to prove the role of individual stress components in energy dissipation, let us assume that:

$$
\sigma_{\mathrm{xa}}=\sigma_{\text {ya }}=\sigma_{\mathrm{za}}=\sigma ; \tau_{\mathrm{xya}}=\tau_{\mathrm{yza}}=\tau_{\mathrm{zxa}}=\tau ; \mathrm{v}=0.3
$$

Eqs (45) and (47) become then:

$$
\phi(T)=\frac{1}{2} \frac{\omega T \sin \alpha}{\sqrt{E^{2}+\eta^{2} \omega^{2}}}\left(\sigma^{2}+2.6 \tau^{2}\right)
$$




$$
\phi(T)=\frac{1}{2} \frac{\omega \mathrm{T} \sin \alpha}{\sqrt{\mathrm{E}^{2}+\eta^{2} \omega^{2}}}\left(1.2 \sigma^{2}+7.8 \tau^{2}\right)
$$

These results suggest to check the following ratios, $\rho_{\mathrm{sn}}$ and $\rho_{\mathrm{dv}}$, of dissipated energies per unit volume:

$$
\rho_{\mathrm{sn}}=\frac{\phi_{\mathrm{s}}(\mathrm{T})}{\phi_{\mathrm{n}}(\mathrm{T})}, \rho_{\mathrm{dv}}=\frac{\phi_{\mathrm{d}}(\mathrm{T})}{\phi_{\mathrm{v}}(\mathrm{T})}
$$

where:

$\varphi_{\mathrm{s}}(\mathrm{T})$ - dissipation energy associated with shear stress components

$\varphi_{\mathrm{n}}(\mathrm{T})$ - dissipation energy associated with normal stress components

$\varphi_{\mathrm{d}}(\mathrm{T})$ - dissipation energy associated with distortions

$\varphi_{v}(\mathrm{~T})$ - dissipation energy associated with volume changes.

At the stress (33), these quantities are given by:

$$
\begin{gathered}
\phi_{\mathrm{s}}(\mathrm{T})=\frac{(1+v) \omega \mathrm{T} \sin \alpha}{\sqrt{\mathrm{E}^{2}+\eta^{2} \omega^{2}}}\left(\tau_{\mathrm{xya}}^{2}+\tau_{\mathrm{yza}}^{2}+\tau_{\mathrm{zxa}}^{2}\right) \\
\phi_{\mathrm{n}}(\mathrm{T})=\frac{\omega \mathrm{T} \sin \alpha}{2 \sqrt{\mathrm{E}^{2}+\eta^{2} \omega^{2}}}\left[\sigma_{\mathrm{xa}}^{2}+\sigma_{\mathrm{ya}}^{2}+\sigma_{\mathrm{za}}^{2}-2 v\left(\sigma_{\mathrm{xa}} \sigma_{\mathrm{ya}}+\sigma_{\mathrm{ya}} \sigma_{\mathrm{za}}+\sigma_{\mathrm{za}} \sigma_{\mathrm{xa}}\right)\right] \\
\phi_{\mathrm{d}}(\mathrm{T})=\frac{(1+v) \omega \mathrm{T} \sin \alpha}{6 \sqrt{\mathrm{E}^{2}+\eta^{2} \omega^{2}}}\left[\left(\sigma_{\mathrm{xa}}-\sigma_{\mathrm{ya}}\right)^{2}+\left(\sigma_{\mathrm{ya}}-\sigma_{\mathrm{za}}\right)^{2}+\left(\sigma_{\mathrm{za}}-\sigma_{\mathrm{xa}}\right)^{2}+6\left(\tau_{\mathrm{xya}}^{2}+\tau_{\mathrm{yza}}^{2}+\tau_{\mathrm{zxa}}^{2}\right)\right] \\
\phi_{\mathrm{v}}(\mathrm{T})=\frac{(1-2 v) \omega \mathrm{T} \sin \alpha}{6 \sqrt{\mathrm{E}^{2}+\eta^{2} \omega^{2}}}\left(\sigma_{\mathrm{xa}}+\sigma_{\mathrm{ya}}+\sigma_{\mathrm{za}}\right)^{2}
\end{gathered}
$$

The values of $\rho_{\mathrm{sn}}$ and $\rho_{\mathrm{dv}}$ vary from:

$$
\rho_{\mathrm{sn}}=2.6\left(\frac{\tau}{\sigma}\right)^{2}, \quad \rho_{\mathrm{dv}}=6.5+19.5\left(\frac{\tau}{\sigma}\right)^{2}
$$

in the case (ii), to:

$$
\rho_{\mathrm{sn}}=\rho_{\mathrm{dv}}=6.5\left(\frac{\tau}{\sigma}\right)^{2}
$$

in the case (i). From comparison of Eqs (iv) and (v) if follows that the role of shear stress components and distortions in energy dissipation is dominant.

\section{THE CASE OF HARMONIC OUT-OF-PHASE STRESS}

Let the stress components in a viscoelastic solid under triaxial harmonic load be:

$$
\sigma_{\mathrm{j}}=\sigma_{\mathrm{ja}} \sin \left(\omega \mathrm{t}+\varphi_{\mathrm{j}}\right) ; \tau_{\mathrm{k}}=\tau_{\mathrm{ka}} \sin \left(\omega \mathrm{t}+\varphi_{\mathrm{k}}\right)
$$

where: $\varphi_{j}(\mathrm{j}=\mathrm{x}, \mathrm{y}, \mathrm{z})$ and $\varphi_{\mathrm{k}}(\mathrm{k}=\mathrm{xy}, \mathrm{yz}, \mathrm{zx})$ are the phase angles. Introducing the vector of complex amplitudes of the stress components:

$$
\bar{\sigma}_{\mathrm{a}}=\left[\bar{\sigma}_{\mathrm{xa}} \bar{\sigma}_{\mathrm{ya}} \bar{\sigma}_{\mathrm{za}} \bar{\tau}_{\mathrm{xya}} \bar{\tau}_{\mathrm{yza}} \bar{\tau}_{\mathrm{zxa}}\right]^{\mathrm{T}}
$$

the vector of the strain components can be written as [2]:

$$
\boldsymbol{\varepsilon}=\mathbf{H} \operatorname{Im}\left[\overline{\boldsymbol{\sigma}}_{\mathrm{a}} \mathrm{e}^{\mathrm{i}(\omega \mathrm{t}-\alpha)}\right]
$$

Here $\mathbf{i}$ is the imaginary unity, Im denotes the imaginary part, and

$$
\begin{gathered}
\bar{\sigma}_{\mathrm{ja}}=\sigma_{\mathrm{ja}} \mathrm{e}^{\mathrm{i} \varphi_{\mathrm{j}}} ; \quad \bar{\tau}_{\mathrm{ka}}=\tau_{\mathrm{ka}} \mathrm{e}^{\mathrm{i} \varphi_{\mathrm{k}}} ; \alpha=\operatorname{arctg} \frac{\eta \omega}{\mathrm{E}} \\
\boldsymbol{\varepsilon}=\left[\varepsilon_{\mathrm{x}} \varepsilon_{\mathrm{y}} \varepsilon_{\mathrm{z}} \gamma_{\mathrm{xy}} \gamma_{\mathrm{yz}} \gamma_{\mathrm{zx}}\right]^{\mathrm{T}}
\end{gathered}
$$

If time-varying stress components act on a viscoelastic solid, the knowledge of resulting strain components enables us to evaluate their combined effect in terms of dissipation energy by means of Eq. (46). In accordance with Eqs (51) through (53), the constitutive equations for strains under the stress state (50) read: 


$$
\begin{aligned}
& \varepsilon_{\mathrm{x}}=\frac{1}{\sqrt{\mathrm{E}^{2}+\eta^{2} \omega^{2}}}\left[\sigma_{\mathrm{xa}} \sin \left(\omega \mathrm{t}+\varphi_{\mathrm{x}}-\alpha\right)-v \sigma_{\mathrm{ya}} \sin \left(\omega \mathrm{t}+\varphi_{\mathrm{y}}-\alpha\right)-v \sigma_{\mathrm{za}} \sin \left(\omega \mathrm{t}+\varphi_{\mathrm{z}}-\alpha\right)\right] \\
& \varepsilon_{\mathrm{y}}=\frac{1}{\sqrt{\mathrm{E}^{2}+\eta^{2} \omega^{2}}}\left[\sigma_{\mathrm{ya}} \sin \left(\omega \mathrm{t}+\varphi_{\mathrm{y}}-\alpha\right)-v \sigma_{\mathrm{xa}} \sin \left(\omega \mathrm{t}+\varphi_{\mathrm{x}}-\alpha\right)-v \sigma_{\mathrm{za}} \sin \left(\omega \mathrm{t}+\varphi_{\mathrm{z}}-\alpha\right)\right] \\
& \varepsilon_{\mathrm{z}}=\frac{1}{\sqrt{\mathrm{E}^{2}+\eta^{2} \omega^{2}}}\left[\sigma_{\mathrm{za}} \sin \left(\omega \mathrm{t}+\varphi_{\mathrm{z}}-\alpha\right)-v \sigma_{\mathrm{xa}} \sin \left(\omega \mathrm{t}+\varphi_{\mathrm{x}}-\alpha\right)-v \sigma_{\mathrm{ya}} \sin \left(\omega \mathrm{t}+\varphi_{\mathrm{y}}-\alpha\right)\right]
\end{aligned}
$$

and

$$
\gamma_{\mathrm{k}}=\frac{2(1+v)}{\sqrt{\mathrm{E}^{2}+\eta^{2} \omega^{2}}} \tau_{\mathrm{ka}} \sin \left(\omega \mathrm{t}+\varphi_{\mathrm{k}}-\alpha\right)
$$

In the present case, Eq. (46) becomes:

$$
\begin{aligned}
& \phi(\mathrm{T})=\frac{\omega}{\sqrt{\mathrm{E}^{2}+\eta^{2} \omega^{2}}} \int_{0}^{\mathrm{T}}\left\{\sigma _ { \mathrm { xa } } \operatorname { s i n } ( \omega \mathrm { t } + \varphi _ { \mathrm { x } } ) \left[\sigma_{\mathrm{xa}} \cos \left(\omega \mathrm{t}+\varphi_{\mathrm{x}}-\alpha\right)-v \sigma_{\mathrm{ya}} \cos \left(\omega \mathrm{t}+\varphi_{\mathrm{y}}-\alpha\right)+\right.\right. \\
& \left.-v \sigma_{\mathrm{za}} \cos \left(\omega \mathrm{t}+\varphi_{\mathrm{z}}-\alpha\right)\right]+\sigma_{\mathrm{ya}} \sin \left(\omega \mathrm{t}+\varphi_{\mathrm{y}}\right)\left[\sigma_{\mathrm{ya}} \cos \left(\omega \mathrm{t}+\varphi_{\mathrm{y}}-\alpha\right)-v \sigma_{\mathrm{xa}} \cos \left(\omega \mathrm{t}+\varphi_{\mathrm{x}}-\alpha\right)+\right. \\
& \left.-v \sigma_{\mathrm{za}} \cos \left(\omega \mathrm{t}+\varphi_{\mathrm{z}}-\alpha\right)\right]+\sigma_{\mathrm{za}} \sin \left(\omega \mathrm{t}+\varphi_{\mathrm{z}}\right)\left[\sigma_{\mathrm{za}} \cos \left(\omega \mathrm{t}+\varphi_{\mathrm{z}}-\alpha\right)-v \sigma_{\mathrm{xa}} \cos \left(\omega \mathrm{t}+\varphi_{\mathrm{x}}-\alpha\right)+\right. \\
& \left.\left.-v \sigma_{\mathrm{ya}} \cos \left(\omega \mathrm{t}+\varphi_{\mathrm{y}}-\alpha\right)\right]+2(1+v) \sum_{\mathrm{k}} \tau_{\mathrm{ka}}^{2} \sin \left(\omega \mathrm{t}+\varphi_{\mathrm{k}}\right) \cos \left(\omega \mathrm{t}+\varphi_{\mathrm{k}}-\alpha\right)\right\} \mathrm{dt}
\end{aligned}
$$

Hence:

$$
\begin{gathered}
\phi(\mathrm{T})=\frac{1}{2} \frac{\omega \mathrm{T} \sin \alpha}{\sqrt{\mathrm{E}^{2}+\eta^{2} \omega^{2}}}\left\{\sigma_{\mathrm{xa}}^{2}+\sigma_{\mathrm{ya}}^{2}+\sigma_{\mathrm{za}}^{2}-2 v\left[\sigma_{\mathrm{xa}} \sigma_{\mathrm{ya}} \cos \left(\varphi_{\mathrm{x}}-\varphi_{\mathrm{y}}\right)+\right.\right. \\
\left.\left.+\sigma_{\mathrm{ya}} \sigma_{\mathrm{za}} \cos \left(\varphi_{\mathrm{y}}-\varphi_{\mathrm{z}}\right)+\sigma_{\mathrm{za}} \sigma_{\mathrm{xa}} \cos \left(\varphi_{\mathrm{z}}-\varphi_{\mathrm{x}}\right)\right]+2(1+\nu)\left(\tau_{\mathrm{xya}}^{2}+\tau_{\mathrm{yza}}^{2}+\tau_{\mathrm{zxa}}^{2}\right)\right\}
\end{gathered}
$$

It is evident that the phase angles of shear stress components do not affect the process of energy dissipation in viscoelastic solids under multiaxial harmonic loads whereas the phase shifts between normal stress components increase the amount of aforementioned energy in comparison with that under in-phase stress. Of course, the amount of dissipated energy depends also on the amplitudes of the stress components and their number, on the load duration and frequency as well as on two parameters of the Kelvin-Voigt's model and Poisson's ratio. The Reader interested in application of three-parameter models of viscoelastic materials is referred, e.g., to $[4,8]$.

\section{DISSIPATION ENERGY IN VISCOELASTIC SOLIDS UNDER MULTIAXIAL PERIODIC LOADS}

In engineering calculations referring to stationary operating conditions, the history of vibratory loads is frequently confined to periodic excitations. In such cases the stress state at a given point can be described by Fourier series representing the normal and shear stress components:

where:

$$
\begin{aligned}
& \sigma_{\mathrm{j}}=\sigma_{\mathrm{j} 0}+\sum_{\mathrm{n}} \sigma_{\mathrm{jn}} \sin \left(\mathrm{n} \omega \mathrm{t}+\varphi_{\mathrm{jn}}\right) \quad ; \mathrm{j}=\mathrm{x}, \mathrm{y}, \mathrm{z} \\
& \tau_{\mathrm{k}}=\tau_{\mathrm{k} 0}+\sum_{\mathrm{n}} \tau_{\mathrm{kn}} \sin \left(\mathrm{n} \omega \mathrm{t}+\varphi_{\mathrm{kn}}\right) \quad ; \mathrm{k}=\mathrm{xy}, \mathrm{yz}, \mathrm{zx}
\end{aligned}
$$

$\sigma_{j 0}, \tau_{\mathrm{k} 0}-$ mean values of the stress components

$\sigma_{\mathrm{jn}}, \tau_{\mathrm{kn}}-$ amplitudes of $\mathrm{n}$-th terms in Fourier expansion of the stress components

$\varphi_{\mathrm{jn}}, \varphi_{\mathrm{kn}}-$ phase angles of $\mathrm{n}$-th terms in Fourier expansion of the stress components

$\omega^{\mathrm{jn}}-$ fundamental circular frequency.

The associated constitutive equation for strains reads [2]:

$$
\boldsymbol{\varepsilon}=\boldsymbol{\varepsilon}_{0}+\sum_{\mathrm{n}} \boldsymbol{H}_{\mathrm{n}} \operatorname{Im}\left[\overline{\boldsymbol{\sigma}}_{\mathrm{n}} \mathrm{e}^{\mathrm{i}\left(\mathrm{n} \omega \mathrm{t}-\alpha_{\mathrm{n}}\right)}\right]
$$

where $\boldsymbol{\varepsilon}$ is given in Eqs (35), $\mathbf{H}_{\mathrm{n}}=\mathbf{H}(\mathrm{n} \omega)$ is obtained from the matrix $\mathbf{H}$ defined in Eqs (35) by inserting n $\omega$ in place of $\omega$, and $\bar{\sigma}_{\mathrm{n}}=\left[\bar{\sigma}_{\mathrm{xn}} \bar{\sigma}_{\mathrm{yn}} \bar{\sigma}_{z \mathrm{n}} \bar{\tau}_{\mathrm{xyn}} \bar{\tau}_{\mathrm{yzn}} \bar{\tau}_{\mathrm{zxn}}\right]^{\mathrm{T}} ; \bar{\sigma}_{\mathrm{jn}}=\sigma_{\mathrm{jn}} \mathrm{e}^{\mathrm{i} \varphi_{\mathrm{jn}}} ; \bar{\tau}_{\mathrm{kn}}=\tau_{\mathrm{kn}} \mathrm{e}^{\mathrm{i} \varphi_{\mathrm{kn}}} ; \alpha_{\mathrm{n}}=\operatorname{arctg} \frac{\eta n \omega}{\mathrm{E}} ; \boldsymbol{\varepsilon}_{0}=\left[\varepsilon_{\mathrm{x} 0} \varepsilon_{\mathrm{y} 0} \varepsilon_{\mathrm{z} 0} \gamma_{\mathrm{xy} 0} \gamma_{\mathrm{yz} 0} \gamma_{\mathrm{zx} 0}\right]^{\mathrm{T}}$ 
The elements of the vector $\boldsymbol{\varepsilon}_{0}$ are given in Eqs (22). Eq. (46) makes it possible to determine the energy dissipation due to the stress components (58) or to any of them. For instance, if a viscoelastic rod is acted upon by the stress:

$$
\sigma_{\mathrm{x}}=\sigma_{\mathrm{x} 0}+\sum_{\mathrm{n}} \sigma_{\mathrm{xn}} \sin \left(\mathrm{n} \omega \mathrm{t}+\varphi_{\mathrm{xn}}\right)
$$

from Eqs (59) and (60) one gets:

$$
\varepsilon_{\mathrm{x}}=\varepsilon_{\mathrm{x} 0}+\sum_{\mathrm{n}} \frac{\sigma_{\mathrm{xn}}}{\sqrt{\mathrm{E}^{2}+\left(\eta \mathrm{n} \omega^{2}\right)}} \sin \left(\mathrm{n} \omega \mathrm{t}+\varphi_{\mathrm{xn}}-\alpha_{\mathrm{n}}\right)
$$

so that:

$$
\dot{\varepsilon}_{\mathrm{x}}=\sum_{\mathrm{n}} \frac{\mathrm{n} \omega \sigma_{\mathrm{xn}}}{\sqrt{\mathrm{E}^{2}+(\eta n \omega)^{2}}} \cos \left(\mathrm{n} \omega \mathrm{t}+\varphi_{\mathrm{xn}}-\alpha_{\mathrm{n}}\right)
$$

For the time period:

$$
\mathrm{T}=\frac{2 \pi}{\omega}
$$

the dissipation energy per unit volume can be calculated as:

$$
\phi(T)=\int_{0}^{T} \sigma_{x} \dot{\varepsilon}_{x} d t=\int_{0}^{T}\left[\sigma_{x 0}+\sum_{n} \sigma_{x n} \sin \left(n \omega t+\varphi_{x n}\right)\right]\left[\sum_{n} \frac{n \omega \sigma_{x n}}{\sqrt{E^{2}+(\eta n \omega)^{2}}} \cos \left(n \omega t+\varphi_{x n}-\alpha_{n}\right)\right] d t
$$

Hence:

$$
\phi(\mathrm{T})=\frac{1}{2} \omega \mathrm{T} \sum_{\mathrm{n}} \frac{\mathrm{n} \sin \alpha_{\mathrm{n}}}{\sqrt{\mathrm{E}^{2}+(\eta n \omega)^{2}}} \sigma_{\mathrm{xn}}^{2}
$$

Similarly, if a viscoelastic solid is subjected to a periodic shear stress:

in conformity with Eqs (59) and (60) the strain response is:

$$
\tau_{\mathrm{xy}}=\tau_{\mathrm{xy} 0}+\sum_{\mathrm{n}} \tau_{\mathrm{xyn}} \sin \left(\mathrm{n} \omega \mathrm{t}+\varphi_{\mathrm{xyn}}\right)
$$

$$
\gamma_{\mathrm{xy}}=\gamma_{\mathrm{xy} 0}+2(1+v) \sum_{\mathrm{n}} \frac{\tau_{\mathrm{xyn}}}{\sqrt{\mathrm{E}^{2}+(\eta n \omega)^{2}}} \sin \left(n \omega t+\varphi_{\mathrm{xyn}}-\alpha_{\mathrm{n}}\right)
$$

Eqs (44), (67) and (68) imply that:

$$
\begin{gathered}
\phi(\mathrm{T})=\int_{0}^{\mathrm{T}} \tau_{\mathrm{xy}} \dot{\gamma}_{\mathrm{xy}} \mathrm{dt}= \\
=\int_{0}^{\mathrm{T}}\left[\tau_{\mathrm{xy} 0}+\sum_{\mathrm{n}} \tau_{\mathrm{xyn}} \sin \left(\mathrm{n} \omega \mathrm{t}+\varphi_{\mathrm{xyn}}\right)\right]\left[2(1+v) \sum_{\mathrm{n}} \frac{\mathrm{n} \omega \tau_{\mathrm{xyn}}}{\sqrt{\mathrm{E}^{2}+(\eta n \omega)^{2}}} \cos \left(\mathrm{n} \omega \mathrm{t}+\varphi_{\mathrm{xyn}}-\alpha_{\mathrm{n}}\right)\right] \mathrm{dt}
\end{gathered}
$$

Eq. (69) yields:

$$
\phi(T)=(1+v) \omega T \sum_{n} \frac{n \sin \alpha_{n}}{\sqrt{E^{2}+(\eta n \omega)^{2}}} \tau_{x y n}^{2}
$$

We now turn to the general state of periodic stress (58). On the basis of Eqs (59) and (60), the following time derivatives of the strain components are obtained:

$$
\begin{gathered}
\dot{\varepsilon}_{\mathrm{x}}=\sum_{\mathrm{n}} \frac{\mathrm{n} \omega}{\sqrt{\mathrm{E}^{2}+(\eta n \omega)^{2}}}\left[\sigma_{\mathrm{xn}} \cos \left(\mathrm{n} \omega \mathrm{t}+\varphi_{\mathrm{xn}}-\alpha_{\mathrm{n}}\right)-v \sigma_{\mathrm{yn}} \cos \left(\mathrm{n} \omega \mathrm{t}+\varphi_{\mathrm{yn}}-\alpha_{\mathrm{n}}\right)-v \sigma_{\mathrm{zn}} \cos \left(\mathrm{n} \omega \mathrm{t}+\varphi_{\mathrm{zn}}-\alpha_{\mathrm{n}}\right)\right] \\
\dot{\varepsilon}_{\mathrm{y}}=\sum_{\mathrm{n}} \frac{\mathrm{n} \omega}{\sqrt{\mathrm{E}^{2}+(\eta n \omega)^{2}}}\left[\sigma_{\mathrm{yn}} \cos \left(\mathrm{n} \omega \mathrm{t}+\varphi_{\mathrm{yn}}-\alpha_{\mathrm{n}}\right)-v \sigma_{\mathrm{xn}} \cos \left(\mathrm{n} \omega \mathrm{t}+\varphi_{\mathrm{xn}}-\alpha_{\mathrm{n}}\right)-v \sigma_{\mathrm{zn}} \cos \left(\mathrm{n} \omega \mathrm{t}+\varphi_{\mathrm{zn}}-\alpha_{\mathrm{n}}\right)\right] \\
\dot{\varepsilon}_{\mathrm{z}}=\sum_{\mathrm{n}} \frac{\mathrm{n} \omega}{\sqrt{\mathrm{E}^{2}+(\eta n \omega)^{2}}}\left[\sigma_{\mathrm{zn}} \cos \left(\mathrm{n} \omega \mathrm{t}+\varphi_{\mathrm{zn}}-\alpha_{\mathrm{n}}\right)-v \sigma_{\mathrm{xn}} \cos \left(\mathrm{n} \omega \mathrm{t}+\varphi_{\mathrm{xn}}-\alpha_{\mathrm{n}}\right)-v \sigma_{\mathrm{yn}} \cos \left(\mathrm{n} \omega \mathrm{t}+\varphi_{\mathrm{yn}}-\alpha_{\mathrm{n}}\right)\right] \\
\dot{\gamma}_{\mathrm{k}}=2(1+v) \sum_{\mathrm{n}} \frac{\mathrm{n} \omega}{\sqrt{\mathrm{E}^{2}+(\eta n \omega)^{2}}} \tau_{\mathrm{kn}} \cos \left(\mathrm{n} \omega \mathrm{t}+\varphi_{\mathrm{kn}}-\alpha_{\mathrm{n}}\right)
\end{gathered}
$$




$$
\begin{aligned}
& \phi(\mathrm{T})=\frac{1}{2} \omega \mathrm{T} \sum_{\mathrm{n}} \frac{\mathrm{n} \sin \alpha_{\mathrm{n}}}{\sqrt{\mathrm{E}^{2}+(\eta n \omega)^{2}}}\left\{\sigma_{\mathrm{xn}}^{2}+\sigma_{\mathrm{yn}}^{2}+\sigma_{\mathrm{zn}}^{2}-2 v\left[\sigma_{\mathrm{xn}} \sigma_{\mathrm{yn}} \cos \left(\varphi_{\mathrm{xn}}-\varphi_{\mathrm{yn}}\right)+\right.\right. \\
& \left.\left.+\sigma_{\mathrm{yn}} \sigma_{\mathrm{zn}} \cos \left(\varphi_{\mathrm{yn}}-\varphi_{\mathrm{zn}}\right)+\sigma_{\mathrm{zn}} \sigma_{\mathrm{xn}} \cos \left(\varphi_{\mathrm{zn}}-\varphi_{\mathrm{xn}}\right)\right]+2(1+v)\left(\tau_{\mathrm{xyn}}^{2}+\tau_{\mathrm{yzn}}^{2}+\tau_{\mathrm{zxn}}^{2}\right)\right\}
\end{aligned}
$$

Eq. (72), as well as other formulae for the amount of energy dissipated in unit volume, may be directly applied to the whole volume if the stress distribution is uniform. Otherwise, in order to determine the total amount of energy dissipated in a given part of the viscoelastic solid, additional calculations are necessary. The example below was chosen not only as an illustration for this problem, but also to gain more information on the influence of shear stress and distortions on energy dissipation in viscoelastic materials.

\section{EXAMPLE}

Compare the total energy dissipated in a viscoelastic rod of constant diameter $2 r_{0}$ and length 1 by its purely axial twisting with that due to its axial tension-compression. Consider the energies dissipated in the rod by its distortions and volume changes.

Solution. In twisting, the shear stress at the radius $r$ is (Fig. 2):

$$
\tau=\frac{\mathrm{r}}{\mathrm{r}_{0}} \tau_{0}
$$

where: $\tau_{0}$ is the shear stress at the outer radius $r_{0}$.

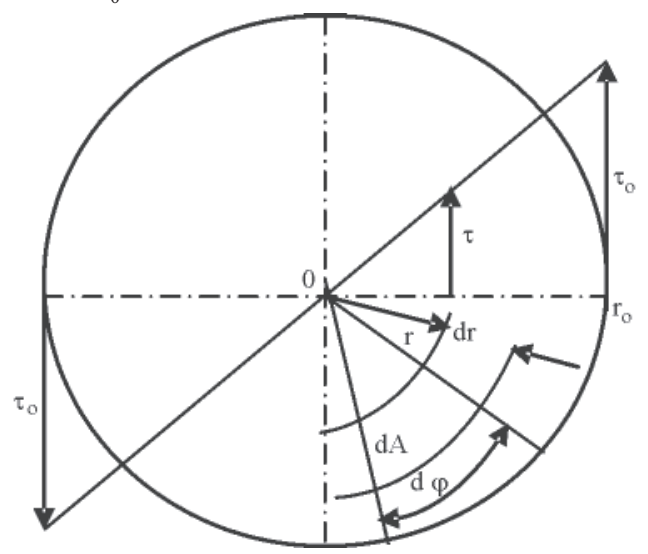

Fig. 2. Distribution of shear stress in the cross-section of a twisted rod

Suppose $\tau_{0}$ is given as:

$$
\tau_{0}=\tau_{0 \mathrm{a}} \sin \omega \mathrm{t}
$$

where: $\tau_{0 \mathrm{a}}$ and $\omega$ are its amplitude and circular frequency. Then:

$$
\tau=\tau_{\mathrm{a}} \sin \omega \mathrm{t}
$$

where:

$$
\tau_{\mathrm{a}}=\frac{\mathrm{r}}{\mathrm{r}_{0}} \tau_{0 \mathrm{a}}
$$

is the stress amplitude at the radius r. For the unit volume:

$$
\mathrm{dV}=\mathrm{dAdl}=\operatorname{rd} \varphi \mathrm{drdl}
$$

we write:

where:

$$
\phi(\mathrm{T})=\phi_{\mathrm{r}}(\mathrm{T})=\int_{0}^{\mathrm{T}} \tau \dot{\gamma} \mathrm{dt}
$$

$$
\gamma=\frac{2(1+v)}{\sqrt{E^{2}+\eta^{2} \omega^{2}}} \tau_{a} \sin (\omega t-\alpha)
$$

is the shear strain at the radius $r$. Thus:

$$
\phi_{\mathrm{r}}(\mathrm{T})=\frac{\mathrm{r}^{2}(1+v) \omega \mathrm{T} \sin \alpha}{\mathrm{r}_{0}^{2} \sqrt{\mathrm{E}^{2}+\eta^{2} \omega^{2}}} \tau_{0 \mathrm{a}}^{2}
$$

For the whole rod, the total energy dissipated by twisting in the time period $\mathrm{T}=2 \pi / \omega, \mathrm{W}_{\mathrm{s}}(\mathrm{T})$, is: 


$$
\begin{aligned}
\mathrm{W}_{\mathrm{s}}(\mathrm{T}) & =\iiint_{\mathrm{V}} \phi_{\mathrm{r}}(\mathrm{T}) \mathrm{dV}=\int_{0}^{2 \pi \mathrm{r}_{0}} \int_{0}^{1} \int_{0} \phi_{\mathrm{r}}(\mathrm{T}) \mathrm{rd} \varphi \mathrm{dr} \mathrm{dl}= \\
& =\frac{(1+\mathrm{v}) \omega \mathrm{T} \sin \alpha}{2 \sqrt{\mathrm{E}^{2}+\eta^{2} \omega^{2}}} \tau_{\mathrm{oa}}^{2} \pi \mathrm{r}_{0}^{2} 1
\end{aligned}
$$

i.e.,

$$
\mathrm{W}_{\mathrm{s}}(\mathrm{T})=\frac{(1+v) \omega \mathrm{T} \sin \alpha}{2 \sqrt{\mathrm{E}^{2}+\eta^{2} \omega^{2}}} \tau_{0 \mathrm{a}}^{2} \mathrm{~V}
$$

where: $\mathrm{V}$ is the volume of the rod. On the other hand, the total energy dissipated in the rod during one cycle of tensioncompression in the case of uniformly distributed harmonic stress of the amplitude $\sigma_{\mathrm{a}}$ and circular frequency $\omega$ amounts to:

$$
\mathrm{W}_{\mathrm{n}}(\mathrm{T})=\frac{\omega \mathrm{T} \sin \alpha}{2 \sqrt{\mathrm{E}^{2}+\eta^{2} \omega^{2}}} \sigma_{\mathrm{a}}^{2} \mathrm{~V}
$$

Through Eqs (73) and (74), the ratio, $\mu_{\mathrm{sn}}$, of dissipated energies in these load cases:

equals:

$$
\mu_{\mathrm{sn}}=\frac{\mathrm{W}_{\mathrm{s}}(\mathrm{T})}{\mathrm{W}_{\mathrm{n}}(\mathrm{T})}
$$

$$
\mu_{\mathrm{sn}}=(1+v)\left(\frac{\tau_{0 \mathrm{a}}}{\sigma_{\mathrm{a}}}\right)^{2}
$$

It means that despite decreasing shear stress towards the rod axis, twisting creates relatively more heat than tensioncompression. Note also that the considered ratio does not depend on the rod dimensions and load frequency.

In the case of unequal frequencies of twisting and tensioncompression, $\omega_{\mathrm{s}} \neq \omega_{\mathrm{n}}$, the ratio $\mu_{\mathrm{sn}}$ depends also on their values and parameters of the Kelvin-Voigt's model. For instance, if:

$$
\frac{\omega_{\mathrm{s}}}{\omega_{\mathrm{n}}}=\frac{\mathrm{m}}{\mathrm{p}}
$$

where: $m$ and $p$ are natural numbers, the ratio of energies dissipated in the rod during the time period:

becomes:

$$
\mathrm{T}_{\mathrm{c}}=\frac{2 \pi \mathrm{m}}{\omega_{\mathrm{s}}}=\frac{2 \pi \mathrm{p}}{\omega_{\mathrm{n}}}
$$

$\mu_{\mathrm{sn}}=\frac{\mathrm{W}_{\mathrm{s}}\left(\mathrm{T}_{\mathrm{c}}\right)}{\mathrm{W}_{\mathrm{n}}\left(\mathrm{T}_{\mathrm{c}}\right)}=(1+v) \frac{\omega_{\mathrm{s}}}{\omega_{\mathrm{n}}} \sqrt{\frac{\mathrm{E}^{2}+\eta^{2} \omega_{\mathrm{n}}^{2}}{\mathrm{E}^{2}+\eta^{2} \omega_{\mathrm{s}}^{2}}}\left(\frac{\tau_{0 \mathrm{a}}}{\sigma_{\mathrm{a}}}\right)^{2}$

Now let us assume that the rod is simultaneously subjected to harmonic twisting and tension-compression of the same frequency. Then, the dissipation energies associated with distortions and volume changes are given per unit volume by Eqs (49) as:

$$
\begin{gathered}
\phi_{\mathrm{d}}(\mathrm{T})=\frac{(1+v) \omega \mathrm{T} \sin \alpha}{6 \sqrt{\mathrm{E}^{2}+\eta^{2} \omega^{2}}}\left(2 \sigma_{\mathrm{a}}^{2}+6 \tau_{\mathrm{a}}^{2}\right) \\
\phi_{\mathrm{v}}(\mathrm{T})=\frac{(1-2 v) \omega \mathrm{T} \sin \alpha}{6 \sqrt{\mathrm{E}^{2}+\eta^{2} \omega^{2}}} \sigma_{\mathrm{a}}^{2}
\end{gathered}
$$

Calculation of the total energy dissipated in the rod by distortions in the time period $\mathrm{T}$ results in:

$$
\mathrm{W}_{\mathrm{d}}(\mathrm{T})=\frac{(1+v) \omega \mathrm{T} \sin \alpha}{6 \sqrt{\mathrm{E}^{2}+\eta^{2} \omega^{2}}}\left(2 \sigma_{\mathrm{a}}^{2}+3 \tau_{0 \mathrm{a}}^{2}\right) \mathrm{V}
$$

For the total energy dissipated in the rod due to volume changes one has:

$$
\mathrm{W}_{\mathrm{v}}(\mathrm{T})=\frac{(1-2 v) \omega \mathrm{T} \sin \alpha}{6 \sqrt{\mathrm{E}^{2}+\eta^{2} \omega^{2}}} \sigma_{\mathrm{a}}^{2} \mathrm{~V}
$$

Their ratio, $\mu_{\mathrm{dv}}$ is:

$$
\mu_{\mathrm{dv}}=\frac{\mathrm{W}_{\mathrm{d}}(\mathrm{T})}{\mathrm{W}_{\mathrm{v}}(\mathrm{T})}=\frac{2(1+\mathrm{v})}{1-2 \mathrm{v}}+\frac{3(1+\mathrm{v})}{1-2 \mathrm{v}}\left(\frac{\tau_{0 \mathrm{a}}}{\sigma_{\mathrm{a}}}\right)^{2}
$$

which again indicates the significance of distortions in energy dissipation.

As far as the elastic strain energy in twisting is concerned, its total maximum value in the rod, $\mathrm{W}_{\mathrm{se}}$, is expressed by:

$\mathrm{W}_{\mathrm{se}}=\frac{1}{2 \mathrm{G}} \iiint_{\mathrm{V}} \tau_{\mathrm{a}}^{2} \mathrm{dV}=\frac{1+\mathrm{v}}{\mathrm{E}} \int_{0}^{2 \pi \mathrm{r}_{0}} \int_{0}^{1} \int_{0}^{1}\left(\frac{\mathrm{r}}{\mathrm{r}_{0}} \tau_{0 \mathrm{a}}\right)^{2} \mathrm{rd} \varphi \mathrm{drdl}$

which gives:

$$
\mathrm{W}_{\mathrm{se}}=\frac{1+\mathrm{v}}{2 \mathrm{E}} \tau_{0 \mathrm{a}}^{2} \mathrm{~V}
$$

The counterpart of this quantity in tension-compression reads:

$$
\mathrm{W}_{\mathrm{ne}}=\frac{1}{2 \mathrm{E}} \sigma_{\mathrm{a}}^{2} \mathrm{~V}
$$

Thus:

$$
\frac{\mathrm{W}_{\mathrm{se}}}{\mathrm{W}_{\mathrm{ne}}}=(1+\mathrm{v})\left(\frac{\tau_{0 \mathrm{a}}}{\sigma_{\mathrm{a}}}\right)^{2}
$$

which corresponds to Eq. (76).

With Eqs (9) and (18), it is easy to prove that the ratio of energy dissipated in the rod after removal of torsional static load to the energy dissipated after removal of axial static load, as well as the ratio of energies dissipated after application of these loads, is also equal to the value of $\mu_{\mathrm{sn}}$ given in Eq. (76).

\section{CONCLUSIONS}

On the basis of three-dimensional constitutive equations for strains in viscoelastic solids [2], the dissipation energy in selected load cases has been determined.

O The dissipation energy in viscoelastic materials is a quadratic form of stress components.

O The time function governing the energy dissipation in homogeneous, isotropic viscoelastic material by normal and shear stress components is the same. Consequently, there is no difference in the time functions governing the dissipation of strain energy of volume changes and the dissipation of strain energy of distortions.

O Under vibratory loads, the dissipation energy rises linearly in course of time and non-linearly with increasing values of the coefficient of viscous damping of the material and load frequency.

O The dissipation energy does not depend on the phase angles of shear stress components but rises with increasing phase shifts between normal stress components of the same frequency. 
In the process of energy dissipation due to normal and shear stress components the role of the latter is dominant.

Comparing the amount of energy dissipated due to distortions with that caused by volume changes it is seen that the influence of distortions on the dissipation effect is much more significant.

\section{BIBLIOGRAPHY}

1. Kolenda J.: Modification of Hooke's law for multiaxial stress in viscoelastic solids. Polish Maritime Research, 2, 2007

2. Kolenda J.: On the behaviour of viscoelastic solids under multiaxial loads. Polish Maritime Research, 3, 2007

3. Lubahn J.D., Felgar R.P.: Plasticity and Creep of Metals. J. Wiley \& Sons, New York, 1961

4. Blake A. (Ed.): Handbook of Mechanics, Materials, and Structures. J. Wiley \& Sons, New York, 1985

5. Nashif A.D., Johnes D.I.G., Henderson J.P.: Vibration Damping. J. Wiley \& Sons, New York, 1985

6. Jakubowicz A., Orłoś Z.: Strength of Materials (in Polish). WNT, Warszawa, 1996.

7. Haslach H.W., Jr., Armstrong R.W.: Deformable Bodies and Their Material Behaviour. J. Wiley \& Sons, 2004

8. Pisarenko G.S., Lebedev A.A.: Resistance of Materials to Deformation and Failure in Complex Stress State (in Russian). Izd. Naukova Dumka, Kiev, 1969

\section{NOMENCLATURE}

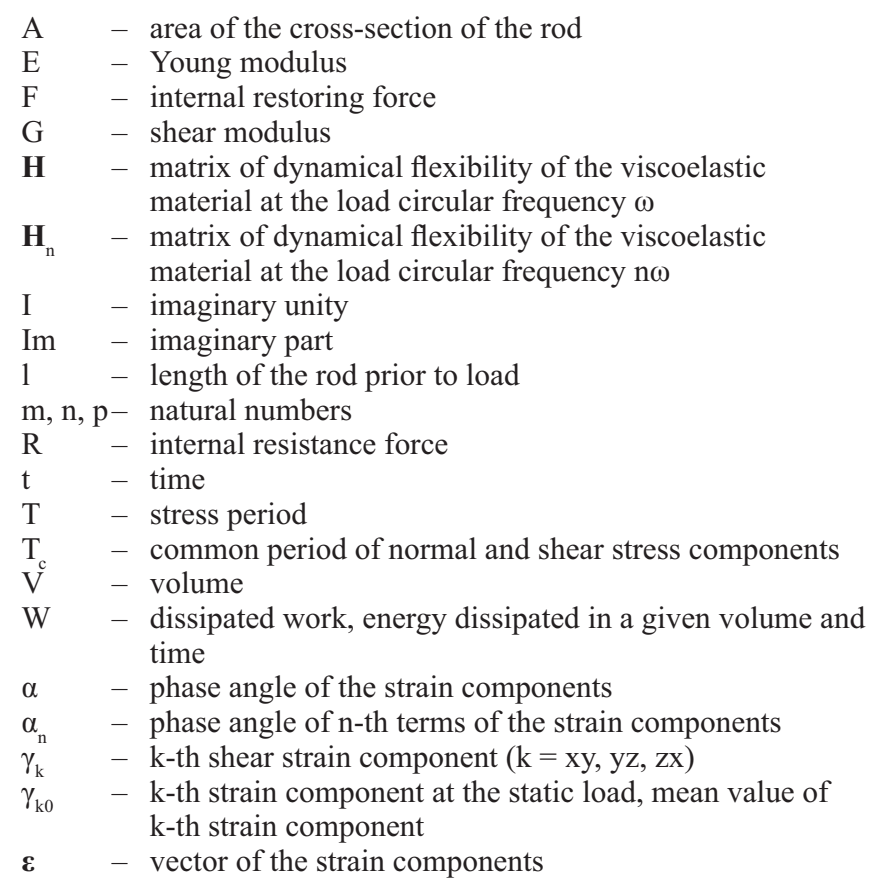

$\varepsilon_{j} \quad-j$-th normal strain component $(j=x, y, z)$

$\varepsilon_{\mathrm{j} 0} \quad-\mathrm{j}$-th strain component at the static load, mean value of $\mathrm{j}$-th strain component

$\boldsymbol{\varepsilon}_{0} \quad-$ vector of mean values of the strain components

$\eta \quad-$ coefficient of viscous damping of normal strain

$\lambda-$ coefficient of viscous damping of shear strain

$\mu_{\mathrm{dv}} \quad-$ ratio of total dissipation energies due to distortions and due to volume changes

$\mu_{\mathrm{sn}} \quad-$ ratio of total dissipation energies due to shear and due to normal stress components

v - Poisson's ratio

$\rho_{\mathrm{dv}} \quad-$ ratio of dissipation energies per unit volume due to distortions and due to volume changes

$\rho_{\mathrm{sn}} \quad-$ ratio of dissipation energies per unit volume due to shear and due to normal stress components

$\sigma_{\mathrm{a}} \quad-$ vector of the amplitudes of stress components

$\sigma_{j} \quad-j$-th stress component

$\sigma_{j a} \quad-$ amplitude of $j$-th stress component

$\sigma_{\text {jn }}-$ amplitude of $n$-th term in Fourier expansion of $\sigma_{j}$

$\sigma_{j 0} \quad-j$-th stress component at the static load, mean value of $\mathrm{j}$-th stress component

$\sigma_{n} \quad-$ vector of complex amplitudes of $n$-th terms in Fourier expansions of the stress components

$\tau_{\mathrm{k}} \quad-\mathrm{k}$-th stress component

$\tau_{\mathrm{ka}} \quad-$ amplitude of $\mathrm{k}$-th stress component

$\tau_{\mathrm{kn}} \quad-$ amplitude of $\mathrm{n}$-th term in Fourier expansion of $\tau_{\mathrm{k}}$

$\tau_{\mathrm{k} 0} \quad-\mathrm{k}$-th stress component at the static load, mean value of $\mathrm{k}$-th stress component

$\varphi_{\mathrm{j}} \quad-$ phase angle of $\mathrm{j}$-th stress component

$\varphi_{\mathrm{jn}} \quad-$ phase angle of $n$-th term in Fourier expansion of $\sigma_{j}$

$\varphi_{\mathrm{k}} \quad-$ phase angle of $\mathrm{k}$-th stress component

$\varphi_{\mathrm{kn}} \quad-$ phase angle of $\mathrm{n}$-th term in Fourier expansion of $\tau_{\mathrm{k}}$

$\varphi \quad-$ dissipation energy per unit volume

$\varphi_{\mathrm{d}} \quad-\quad$ dissipation energy associated with distortions

$\varphi_{\mathrm{n}} \quad-$ dissipation energy associated with normal stress components

$\varphi_{\mathrm{s}} \quad-$ dissipation energy associated with shear stress components

$\varphi_{\mathrm{v}} \quad-$ dissipation energy associated with volume changes

$\psi_{0} \quad-$ elastic strain energy per unit volume under static load

$\omega \quad-$ circular frequency, fundamental circular frequency of periodic stress

$\omega_{\mathrm{s}} \quad-$ circular frequency of twisting

$\omega_{\mathrm{n}} \quad-$ circular frequency of tension-compression

$(\bar{n}) \quad-$ complex quantity

\section{CONTACT WITH THE AUTHOR}

Prof. Janusz Kolenda

Mechanic-Electric Faculty,

Polish Naval Academy

Śmidowicza 69

81-103 Gdynia POLAND

phone : +48 586262789 